\title{
Neuroimaging Biomarkers for Psychosis
}

\author{
Brandon M. Hager • Matcheri S. Keshavan
}

Published online: 6 March 2015

(C) Springer International Publishing AG 2015

\begin{abstract}
Background Biomarkers provide clinicians with a predictable model for the diagnosis, treatment, and follow-up of medical ailments. Psychiatry has lagged behind other areas of medicine in the identification of biomarkers for clinical diagnosis and treatment. In this review, we investigated the current state of neuroimaging as it pertains to biomarkers for psychosis. Methods We reviewed systematic reviews and meta-analyses of the structural (sMRI), functional (fMRI), diffusion tensor (DTI), positron emission tomography (PET), and spectroscopy (MRS) studies of subjects at risk or those with an established schizophrenic illness. Only articles reporting effect sizes and confidence intervals were included in an assessment of robustness.

Results Out of the identified meta-analyses and systematic reviews, 21 studies met the inclusion criteria for assessment. There were 13 sMRI, 4 PET, 3 MRS, and 1 DTI studies. The search terms included in the current review encompassed familial high risk (FHR), clinical high risk (CHR), first episode (FES), chronic (CSZ), schizophrenia spectrum disorders (SSDs), and healthy controls (HCs).

Conclusions Currently, few neuroimaging biomarkers can be considered ready for diagnostic use in patients with psychosis. At least in part, this may be related to the challenges inherent in the current symptom-based approach to classifying these disorders. While available studies suggest a possible value of
\end{abstract}

This article is part of the Topical Collection on Psychosis

B. M. Hager $\cdot$ M. S. Keshavan $(\bowtie)$

Massachusetts Mental Health Center Division of Public Psychiatry, Beth Israel Deaconess Medical Center, Department of Psychiatry, Harvard Medical School, 75 Fenwood Road, 5th Floor, Boston, MA 02115, USA

e-mail: mkeshava@bidmc.harvard.edu

B. M. Hager

e-mail: bhager@bidmc.harvard.edu imaging biomarkers for monitoring disease progression, more systematic research is needed. To date, the best value of imaging data in psychoses has been to shed light on questions of disease pathophysiology, especially through the characterization of endophenotypes.

Keywords Neuroimaging biomarkers · Psychosis MRI · fMRI $\cdot$ PET $\cdot$ MRS

\section{Introduction}

Recent decades have witnessed major advances in understanding of the neurobiological basis of mental functions in health and disease, as well as an expanding basis of genetic and environmental etiology of mental disorders. Such knowledge has led to the hope that we will have an array of biomarkers of clinical value in psychiatry. A biomarker is a characteristic that is objectively measured and evaluated as an indicator of normal biologic processes, pathologic processes, or biological responses to a therapeutic intervention [1]. Biomarkers can provide clinicians with a universal, predictable model for the diagnosis, treatment, and follow-up of medical ailments. However, there are few laboratory tests in psychiatry other than those used to rule out primary medical illnesses (e.g., hypothyroidism in depression). In a recent analysis of over 3200 studies investigating biomarkers (including imaging) using a rigorous measure of quality of evidence, only one study passed an a priori threshold of clinical applicability $[2 \bullet \bullet$. In a nutshell, psychiatry has lagged behind other areas of medicine in the identification of biomarkers for clinical diagnosis and treatment.

One wonders why. Obvious challenges include the relative inaccessibility of brain tissue for study, scarcity of valid animal models, and the substantive symptomatic, biological, and 
etiological heterogeneity of psychiatric illnesses [3]. There is too much cross-disorder overlap and too much withindisorder variability. The field may have focused for too long on theories that are difficult to test. While a vast literature has accumulated on biomarkers of interest, most results are of small effect sizes, use single biomarker studies, and robust replications are rare; other problems have included publication bias, inconsistent terminology, and incomplete reporting [2]. Clearly, there is a need to take stock of our current approaches to biomarker identification and testing and identify more fruitful ways forward (Table 1).

A great deal of knowledge on the neurobiological basis of psychotic disorders has accrued in recent years with the advent of increasingly sophisticated brain imaging techniques. In this review, we sought to cull the extant neuroimaging literature in psychotic disorders to address the above questions, while assessing the results on the dimensions of methodological quality, strength, replicability, and consistency as potential biomarkers for psychotic disorders. We examined the applicability of imaging biomarkers for the purposes of diagnosis (i.e., distinguishing between those with disease and healthy subjects and between diagnoses), prediction of outcome/treatment response, and elucidation of pathophysiology. We chose to focus on effect sizes and their confidence intervals (CI) for determining whether a difference is large enough to be important. Replicability in the context of this review is the precision with which we can reproduce a given effect size based on the $95 \%$ CI. The CI describes the uncertainty inherent in the effect size point estimate and describes a range of values within which we can be reasonably sure that the true effect actually lies [4]. The combined use of an effect size and its $\mathrm{CI}$ enables one to assess the relationships within data more effectively than the use of $p$ values, regardless of statistical significance [5]. There is considerable variability between neuroimaging studies, which has hindered progress toward identifying biomarkers [6].
Our aim was to integrate available information from metaanalyses and systematic reviews of several neuroimaging paradigms, to then determine the potential value of neuroimaging biomarkers for diagnosis, longitudinal monitoring, and pathophysiological research in psychosis. We reviewed studies of structural magnetic resonance imaging (sMRI), functional magnetic resonance imaging (fMRI), magnetic resonance spectroscopy (MRS), diffusion tensor imaging (DTI), and positron emission tomography (PET)/single photon emission computed tomography (SPECT). We chose a meta-review approach to allow for the assessment of the strength of the evidence and the consistency of findings across reviews [7•].

\section{Methods}

We searched PUBMED and Google Scholar for metaanalyses and systematic reviews of psychotic populations that used neuroimaging as the method of measurement. We used the search terms, "Schizophrenia," "Effect size," "Meta-analysis," "Systematic review," "Neuroimaging," "Psychosis," "Biomarker," "First episode," "High risk," "MRI," "fMRI," "DTI," "PET," "MRS" and "1-H MRS", "Structural," "Functional," "Metabolism," "Structure," "Function," and "Metabolic" in several combinations. Studies were then selected for inclusion in a quality assessment based on whether the results included summary effect sizes and tests for heterogeneity and publication bias. Effect sizes are a useful tool to integrate the findings of studies that utilized different neuroimaging methods. If a difference exists in a population, the effect size can be used to determine whether the result warrants further study and whether the difference is large enough to be important. The specific populations included in this review are chronic schizophrenia (CSZ) with some instances of schizophrenia spectrum disorders (SSDs) included in the sample, first-episode schizophrenia (FES), bipolar disorder with

Table 1 Road blocks to biomarker development in psychiatry and potential strategies to circumvent them

\begin{tabular}{|c|c|}
\hline Road blocks & Potential strategies \\
\hline Symptom-based diagnoses are the gold standard for validation & $\begin{array}{l}\text { Develop and test neuroscience-based disease categories for biomarker } \\
\text { validation }\end{array}$ \\
\hline $\begin{array}{l}\text { Use of small sample sizes, low power, and frequent non-replication; } \\
\text { use of single imaging domains, single biomarkers of small effect }\end{array}$ & $\begin{array}{l}\text { Multisite studies, data sharing; multimodal imaging studies, multivariate } \\
\text { biomarker studies, use of "omic" data (such as genome-wide, } \\
\text { transcriptome, and proteome data) and pattern classification algorithms }\end{array}$ \\
\hline $\begin{array}{l}\text { Difficulty testing and developing animal models for complex, } \\
\text { multidimensional psychopathological syndromes with } \\
\text { multifactorial causation }\end{array}$ & $\begin{array}{l}\text { Testing biomarkers in conditional knockout models; task-independent } \\
\text { fMRI studies; translational cognitive domains for testing in animal } \\
\text { models }\end{array}$ \\
\hline $\begin{array}{l}\text { Publication bias, inconsistent terminology and incomplete } \\
\text { reporting }\end{array}$ & $\begin{array}{l}\text { Pre-registration of imaging biomarker studies; standard framework } \\
\text { for reporting and evaluating results }\end{array}$ \\
\hline $\begin{array}{l}\text { Poor reliability, stability, replicability, and high measurement } \\
\text { variability }\end{array}$ & $\begin{array}{l}\text { Wider use of high field scanners; automated assessments of imaging } \\
\text { data; uniform approaches to data analyses }\end{array}$ \\
\hline
\end{tabular}


psychotic features (BPD), clinical high risk (CHR), and familial high risk (FHR).

\section{Exclusion Criteria}

The initial search yielded several hundred results, many of which were excluded based on reading the title and abstract. Of the 56 articles that survived the initial search, several were excluded from the results assessments for meeting the following criteria: absence of summary effect sizes, results that were reported as significant for heterogeneity, as measured by Cochrane's $Q$ statistic [11], and results that were significant for publication bias with metrics like Egger's $p$ [14] were not included. Publication bias arises when studies with statistically significant results are more likely to be published and cited. Studies published before the year 2000 were excluded to reduce the likelihood of study overlap in the meta-analyses. Voxel-based imaging studies were not included, as voxelbased studies do not allow for the estimation of effect sizes. Additionally, voxel-based studies present difficulty in analysis owing to differences across studies in smoothing kernel size, slice thickness, statistical threshold, and whether Jacobian modulation is used in the pre-processing [8]. A list of the studies excluded from this review will be available upon request.

\section{Study Quality}

For studies that met the criteria for the assessment of strength, consistency and replicability, the assessment of review methodological quality was guided by the Assessing the Methodological Quality of "Systematic Reviews (AMSTAR) Checklist, a validated tool for assessing the methodological quality of systematic reviews and meta-analyses" $[9,10]$.

\section{Data Extraction}

The data were extracted by one author (B.H.) and checked twice additionally to assure accuracy. In case of uncertainties, data were checked by another author (M.K.). Similarity vs. variance (consistency) in the direction of the effect sizes was quantified using $I$-squared $\left(I^{2}\right)$ [11]. In instances where it was not reported, we calculated $I^{2}$ using the following formula: $I^{2}=Q-(n-1) / Q$. Neuroimaging studies that included effect sizes were assessed for robustness on four dimensions. (1) Quality of the study was assessed using the AMSTAR rating system where ratings below $27 \%$ were considered low quality, $27-73 \%$ moderate quality, and $73-100 \%$ high quality. (2) The magnitude (strength) of the effect was determined according to Cohen's $d$ [12], where an effect size of \pm 0.20 indicates a small effect; \pm 0.50 is a medium effect; and \pm 0.80 a large effect. We only reported on results whose effect sizes were $\geq 0.20$ or $\leq-0.20$. Hedge's $g$ [13], another measure of effect size, was used in certain studies to correct for bias due to smaller sample sizes, none of which are included in Table 1. (3) Replicability (the precision of the effect size estimate) was determined by the $95 \% \mathrm{CI}$ of the reported effect sizes. We decided to only include results in which the endpoints of the CI were not close to zero. As a means of determining endpoints that were not close to zero, we used a cutoff point of Cohen's measure for a small effect $( \pm 0.20)$ as the lower limit for a strong and reproducible effect. The cutoff point was used to narrow the reportable data and hopefully provide CIs of smaller width. (4) Consistency (heterogeneity across the pooled study results) was measured by $I^{2}$, with less than $25 \%$ considered to have negligible heterogeneity, between 25 and $75 \%$ moderate heterogeneity, and greater than $75 \%$ might represent substantial heterogeneity [11]. Due to the disproportionate amount of sMRI studies, we created Table 1 for a focused assessment of the robustness of the structural results

\section{Results}

After the exclusion of several studies due to the lack of available information, a total of 21 studies met the criteria for assessment, with 13 sMRI studies ( 9 cross-sectional, 4 longitudinal), 4 PET/SPECT studies, 3 MRS studies, and 1 DTI study that reported effect sizes. While several fMRI metaanalyses and systematic reviews have been conducted, due to the use of voxel-based analyses, effect sizes were not reported, making the data incomparable to other neuroimaging measures, and therefore, we could not report the specific quantitative results in this review. sMRI studies provided the largest amount of results that fit the inclusion criteria, which are enumerated in Table 2. Studies with other modalities had either insufficient data, were underpowered, or exhibited significant levels of heterogeneity that would prevent an accurate estimate of effect size across studies. We herein chose to focus on sMRI studies and will briefly review the extant literature on other imaging modalities.

\section{Cross-sectional sMRI Studies in Schizophrenia Versus Healthy Controls}

Studies of populations of medicated patients with schizophrenia, mostly chronic, have shown a large number of structural alterations that showed small to moderate effect sizes, with strong replicability and consistency across studies. While some study overlap was present due to the large amalgamation of structural studies across multiple phases of SZ by Hajima et al. [15], we chose to include certain studies, despite overlap, due to their focus on specific phases of SZ. The review by Haijma and colleagues provided the most robust information regarding structural abnormalities due to the influence of antipsychotic (AP) treatment. The most robust findings in medicated CSZ/SSD were volume increases in the third and lateral 


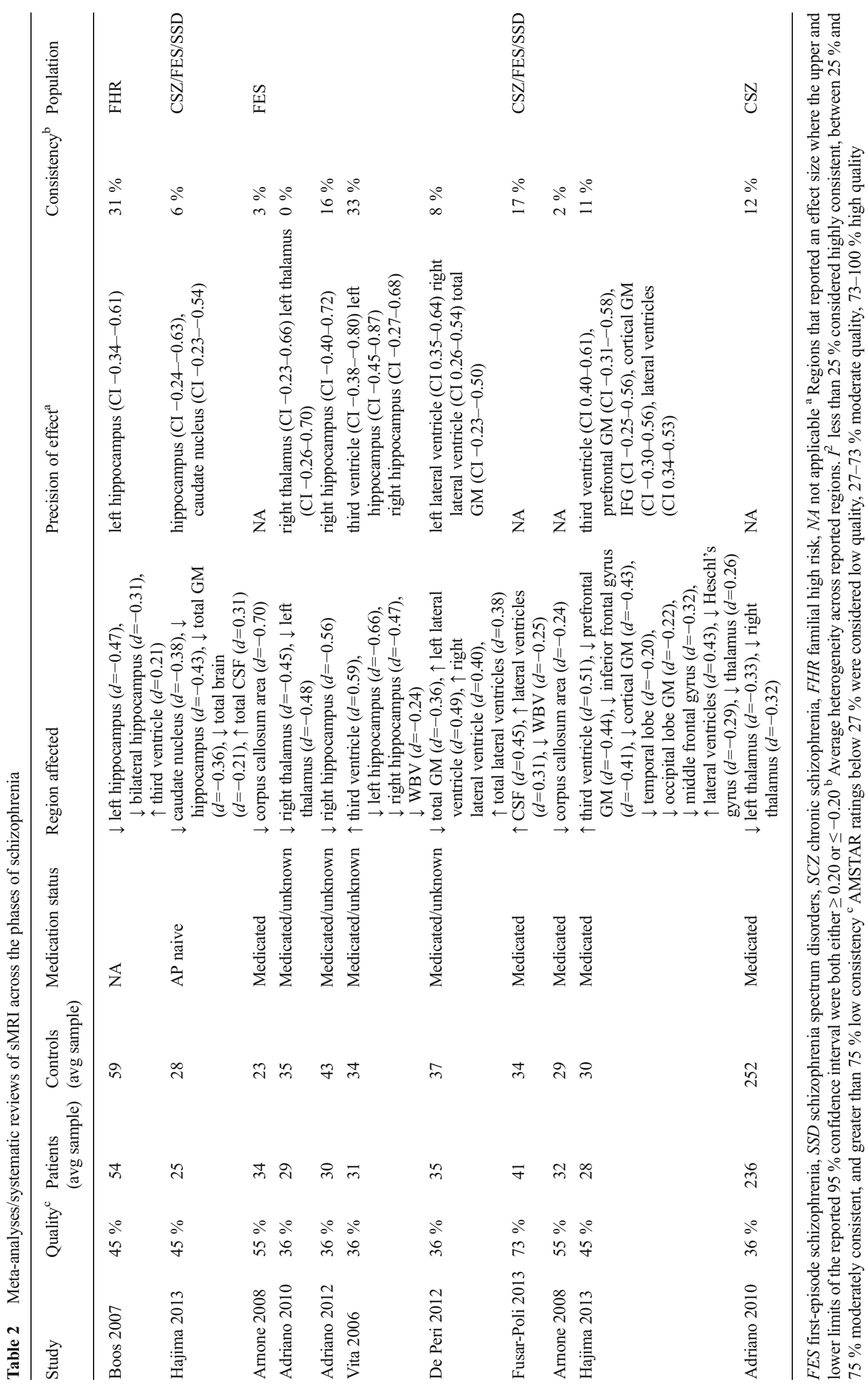


ventricles. Conversely, volume decreases in cortical gray matter (GM), prefrontal GM, and the inferior frontal gyrus were observed.

Several systematic reviews and meta-analyses have investigated FES patients. In the analysis by Adriano et al. [16], medicated and unmedicated FES patients showed a decrease in right and left thalamus volumes. Haijma et al.'s review which included Adriano's findings [15] along with data from CSZ and SSD patients showed thalamic volume decrease overall with a small effect size. In a subsequent analysis of FES, Adriano et al. [17] found a significant reduction in right hippocampal volume relative to controls. Vita [18] showed a robust increase in right lateral ventricle volume in FES patients. De Peri et al. [19] found a reduction in total GM and increases in both the right, left, and total lateral ventricle volumes. Relatively few meta-analyses have reported sMRI data in AP naïve patients with CSZ/FES/SSD, with the most robust findings being volume reductions in the caudate and hippocampus [15]. Interestingly, in FHR, Boos et al. [20] observed a reduction in the volume of the left hippocampus.

A key question in ascertaining the diagnostic value of a biomarker is whether it differs between the population of interest and another diagnostic category. Most studies that exist have used bipolar disorder as a comparator. When comparing BPD and CSZ [21], a reduced right amygdala volume was seen in CSZ with a low to moderate effect size. Less consistent findings were larger increases in left and right lateral ventricle volumes in CSZ and more prominent reductions in left caudate volume. Additionally, Kempton et al. (2008) showed an increase in lateral ventricle volume $(d=-0.39$; CI $0.24-0.55$; $\left.I^{2}=19 \%\right)$ and a decreased corpus callosum area $(d=-0.43$; CI $-0.12-0.74 ; I^{2}=0 \%$ ) [22], with a reduced corpus callosum area also reported in FES and SCZ subjects by Arnone et al. [23]. A study of first-episode BPD and FES subjects [19] found shared abnormalities of increased lateral ventricle volume and decreased total GM volume, with more pronounced differences in FES. There is evidence for a large degree of overlap between CSZ/SSD and BPD in large-scale studies such as BSNIP [24].

\section{Longitudinal sMRI Studies}

Due to variability in the stage of illness, diagnostic criteria, treatment regimen, and image acquisition, it is difficult to identify a specific link between the differences in structure and a biomarker(s) for psychosis. To minimize the variability inherent in the cross-sectional results, longitudinal studies provide more detail in assessing viable biomarker candidates. Certain structural differences that occur in schizophrenia are shown to be progressive over time.

Longitudinal meta-analyses provide an illustration of how certain structural differences that are present in schizophrenic patients at the onset of psychosis undergo a progressive degeneration. Over time, FES subjects showed a decrease in frontal $\left(d=-0.39 ; \mathrm{CI}-0.22-0.57 ; I^{2}=0 \%\right)$ and parietal $(d=$ -0.30 ; CI $-0.12-0.48 ; I^{2}=0 \%$ GM, and a pooled sample of CSZ and FES subjects showed reductions in the right posterior superior temporal gyrus (rSTG) $(d=-0.62$; CI -0.32 $\left.0.92 ; I^{2}=39 \%\right)$ and planum temporale $(d=-0.37$; CI 0.02 $0.76 ; I^{2}=0 \%$ ) [25]. Olabi et al. showed that CSZ subjects had decreases in frontal $\left(d=-0.51 ;\right.$ CI $\left.-0.26-0.76 ; I^{2}=0 \%\right)$, parietal $\left(d=-0.53\right.$; CI $\left.-0.23-0.84 ; I^{2}=4 \%\right)$, and temporal lobe $\left(d=-0.49 ; \mathrm{CI}-0.21-0.76 ; I^{2}=0 \%\right) \mathrm{WM}$, and frontal lobe tissue $\left(d=-0.48 ; \mathrm{CI}-0.18-0.78 ; I^{2}=0 \%\right)$ volumes [26]. Olabi et al. also showed a progressive increase in right lateral ventricle volume, and Fusar-Poli et al. [27] and Kempton et al. [28] showed an increase in bi-lateral lateral ventricle volume in CSZ, but these results did not meet the criterion for replicability and/or consistency.

\section{Other Neuroimaging Modalities}

Among the other imaging modalities, PET studies were noteworthy. The meta-analysis by Fusar-Poli [29] showed that CSZ/SSD patients have an increase in striatal dopamine synthesis capacity ( $g=0.87$, CI $1.194-0.594)$. Howes [30] found that presynaptic dopaminergic function was increased in CSZ/ SSD whether exposed to AP medication $(d=0.69)$ or not $(d=$ 0.67 ), but these results were significant for heterogeneity. A substantive body of research has accumulated on MRS studies of $N$-acetylaspartate (NAA) and glutamate (glu) concentrations in CSZ, CHR, FHR, and FES. Kraguljac et al. [31] showed reductions of NAA in the frontal lobe $(d=-0.44$; CI $-0.23-0.65)$ and basal ganglia $(d=-0.22$; CI $-0.05-0.48)$ for CSZ and FES subjects. Brugger [32] showed reductions of NAA in the thalamus for FES $\left(d=-0.40 ; \mathrm{CI}-0.06--0.75 ; I^{2}=\right.$ $23 \%)$ and SCZ $\left(d=-0.32\right.$; CI $\left.-0.10-0.53 ; I^{2}=26 \%\right)$ subjects. Marsman [33] found a decrease in glu $(d=-0.39)$ and increase in glutamine $(\mathrm{gln})(d=0.40)$ in frontal regions in FES and CSZ patients compared with HC. As gln is a potential marker of glutamatergic neurotransmission, this finding suggests increased glutamate turnover in the frontal brain regions. Marsman et al. performed group-by-age associations revealing "that in patients with schizophrenia, glutamate and glutamine concentrations decreased at a faster rate with age as compared with healthy controls." In both CHR and FHR subjects, Brugger found NAA reductions in the temporal lobe $\left(d=-0.38\right.$; CI $\left.0.03-0.79 ; I^{2}=44 \%\right)$ and thalamus $(d=$ $-0.72 ; I^{2}=0 \%$ ), while Mondino et al. [34], showed significantly lower NAA/Cr level in the prefrontal cortex $(g=-0.42$; CI $-0.23-0.61)$, though heterogeneity was not assessed. The $\mathrm{NAA} / \mathrm{Cr}$ reduction was only significant for patients who had not passed the peak age of risk for schizophrenia.

We were able to find one meta-analysis [35] utilizing DTI to investigate the splenium and genu of the corpus callosum, which reported effect sizes. The meta-analysis of the genu, 
showing lower fractional anisotropy (FA), yielded a low effect size ( $d=0.223$; CI $-0.08-0.53)$, and the analysis investigating the splenium, also showing lower FA, yielded a modest effect size of ( $d=0.527$; CI $0.22-0.84)$ but with significant heterogeneity. The results may indicate that patients had lower FA in these regions compared to healthy volunteers; however, there is too much inconsistency between studies. Ellison-Wright et al. [36] conducted an activation likelihood estimation meta-analysis (a technique for coordinate-based meta-analysis of neuroimaging data) finding that "over all studies, significant reductions were present in the left frontal deep white matter and the left temporal deep white matter. The region in the left frontal lobe is traversed by white matter tracts interconnecting the frontal lobe, thalamus, and cingulate gyrus. The second region, in the temporal lobe, is traversed by white matter tracts interconnecting the frontal lobe, insula, hippocampus-amygdala, temporal, and occipital lobe." A recent meta-analysis on FES [37] showed that "disruptions of white matter integrity were found in the cortical, subcortical brain regions and white matter associative and commissural tracts, suggesting that changes of cortical-subcortical white matter integrity were found at an early stage of the disorder."

Due to the high variability in functional imaging task paradigms, there is a great deal of systematic reviews and metaanalyses of working memory, executive function, emotion recognition, and other task-based activation studies. Multiple meta-analyses of brain function have found differences in activity in the prefrontal cortex (PFC) [27, 38-42], anterior cingulate cortex (ACC) [38, 41, 44], insula [40, 44, 45], thalamus $[38,39,43]$, and superior temporal gyrus $(40-41,44,46)$. In a recent study [40], comparing CHR and FHR subjects, vulnerability to psychosis was associated with prefrontal, cingulate, and middle temporal abnormalities in both groups, with CHR subjects having additional abnormalities in the parietal lobe, superior temporal lobe, and insula. When looking at differences between those that did and did not transition to psychosis, differences in prefrontal, hippocampal, and striatal components were found to be more pronounced in CHR subjects.

\section{Multimodal Imaging}

Smieskova et al. performed an analysis of multimodal studies, finding that psychotic symptoms displayed in altered prefrontal and hippocampal activations were associated with striatal dopamine and thalamic glutamate functions [40]. Another multimodal analysis conducted by Cooper et al. found that FHR subjects had decreased grey matter with hyperactivation in the left inferior frontal gyrus/amygdala and decreased grey matter with hypo-activation in the thalamus [43]. fMRI alterations were found in the right temporal lobe, left thalamus, and left cerebellum as neurofunctional correlates of familial risk for schizophrenia. The combined structural and functional multimodal analysis identified abnormalities within the left inferior frontal gyrus, amygdala, and left thalamus. Radua et al. conducted a multimodal study of FES frontal/ anterior cingulate cortices, and in the bilateral insulae, where patients showed a decrease in grey matter volume as well as abnormal functional response [44]. They conceded, however, that some of these changes may be partially related to treatment with antipsychotic medication. A high-quality longitudinal fMRI study of brain function in CHR subjects showed that reduced prefrontal activation during a working memory task was associated with a reduction in gray matter volume in the same area [46]. Changes in anterior cingulate activation were correlated with functional improvement in CHR patients, consistent with the role of this region in multiple cognitive and social processes.

\section{Discussion}

Imaging Biomarker Studies in Psychotic Disorders: What Have We Learned?

In this paper, a limited attempt was made to review the voluminous literature on neuroimaging biomarkers in psychotic disorders. We chose to only include meta-analyses and systematic reviews in the assessment of potential biomarkers for psychosis, which means that some incidental findings might have been left out. However, it is safe to conclude that while many structural, functional, and neurochemical differences are seen between patients with schizophrenia and healthy controls, only a few such changes are robust and consistent. Further, it is unclear whether any of these biomarkers can help reliably distinguish between SZ and other psychiatric disorders. In particular, the spectrum of psychotic disorders such as SZ, schizoaffective, and psychotic bipolar disorders appear to show more of a continuum of symptomatology, cognitive, electrophysiological, and neurobiological [47] alterations rather than clear-cut distinctions across categories. This suggests that brain-based biomarkers may not exactly obey the boundaries set by symptom constellations such as the Diagnostic and Statistical Manual (DSM) categories. Previous attempts to validate biomarkers using DSM diagnoses as the gold standard may have been flawed [48].

By contrast, with the rather limited ability of imaging biomarkers to distinguish disorder categories, evidence for differences in brain structure across the different phases of a psychotic illness seem somewhat more promising; such information may be of value for the purposes of outcome prediction, longitudinal monitoring, and phase-specific treatment planning. Significant differences in brain structure have been shown across multiple phases of SZ (premorbid, prodromal, FES, and CSZ) [49]. A limited literature exists which indicates progression of brain structural changes, both global and regional, within the same individuals over time. While subtle 
brain structure alterations, particularly in frontotemporal regions, are already seen in FHR adolescents [50], cortical gray matter thickness reductions and third ventricle enlargement appear to characterize the transition from the prodrome to the first psychotic episode [51]. Interestingly, relatives who do not progress to psychosis appear to even have a reversal of their brain structure alterations [52]. During the early course of SZ, there appears to be further progressive reduction of superior temporal cortices [25]. Enlarged lateral ventricles, which are seen consistently in CSZ, are not a prominent feature of FES. Overall, the heterogeneity of outcome of schizophrenia suggests that the neuropathology of the illness may not necessarily progress and may even be reversed with appropriate intervention and/ or resilience factors supervene; this has led to the suggestion that a staging model of schizophrenia may be a better way to conceptualize the natural course of this illness [53•].

The most important insights that have emerged from neuroimaging studies of psychoses thus far have been in our understanding of pathophysiology. Some observations using PET, such as the increase in presynaptic dopamine synthesis capacity and function in the striatum, have reignited interest in the decade-old dopamine hypothesis of schizophrenia [54]. MRS studies showing alterations in glutamatergic metabolites, albeit less consistent, have generated renewed interest in the glutamate hypothesis [33].

Another important direction in research is to examine the putative links between genetic etiology and phenotypic alterations as assessed by imaging studies. Biomarkers that have a strong genetic basis are trait-related and differ between unaffected relatives and healthy subjects are called endo- or (intermediate) phenotypes and are a valuable step in efforts to identify genetic etiology [55]. Imaging measures, relative to other biomarkers, can be reliably measured, are heritable, and provide large effect sizes, making them good candidates for endophenotypes for psychiatric genetics research [56]. However, given the complex, polygenetic etiology of psychiatric disorders, large-scale multivariate analyses are needed. A recent initiative is the Enhancing NeuroImaging Genetics through Meta-Analysis (ENIGMA) Consortium, a worldwide consortium of over 70 large-scale investigations that integrate imaging data of over 12,826 subjects. One of the first studies in the ENIGMA study was to identify gene variants associated with hippocampal volume using a genome-wide association study [57••]. In recent years, there is an increasing emphasis on investigating interconnected neural systems as a way to better understand pathophysiology. The human connectome project [58•] seeks to systematically map brain macrocircuits and their relationship to behavior in large samples of adults in health and disease. Connectome-based analyses are beginning to reveal large-scale disruptions in interregional brain connectivity in schizophrenia [59•]. Taken together, recent advances in imaging technologies are raising the hope that understanding of the etiopathology of psychoses will rapidly expand in the coming years.

Imaging Biomarkers in Psychoses: Strategies for the Future

It is clear from the above that while biomarker studies have advanced our understanding of the pathophysiology of psychotic disorders, few actionable biomarkers are ready for clinical use. How do we move forward? First, an alternative, perhaps more fruitful approach to diagnosis, might be to eschew our efforts to develop diagnostic markers across symptombased categories but begin to classify diseases based on dimensional biomarkers, which can then be validated by other biomarkers (not used to derive the classification in the first place), treatment response, as well as etiological measures such as genomic data $[47,60 \bullet$, Clementz et al. under review]. For a medical analogy, think of a time when people with cough and chest pains were classified into those with and without breathlessness or productive cough. An effort to validate sputum examination for Bacilli as a diagnostic test between these two groups will clearly fail but more likely to succeed if the classification was between cough and chest pain patients with vs without a lesion in a chest X-ray.

Second, developing and testing multimodal imaging methodologies are of value. Different imaging modalities offer complementary information about altered neurobiology; for this reason, it is of value to use multimodal imaging data for understanding pathophysiology as well as potential clinical use. For example, in Alzheimers disease, combined use of structural MRI (for hippocampal volumes), fluorodeoxyglucose (FDG) PET (for regional cerebral metabolism), and PET using ${ }^{11} \mathrm{C}$-labeled tracer Pittsburgh compound $\mathrm{B}$ (PIB) radioligands (for imaging amyloid plaques) has been proposed [61•]. In psychotic disorders, multimodal imaging studies have often combined multiple MRI approaches, i.e., sMRI, fMRI, and DTI [62•]. In a multimodal imaging study in prodromal patients, Fusar-Poli et al. showed that subcortical dopamine synthesis as measured by PET was negatively correlated with prefrontal function as measured by fMRI with a working memory task [54].

Third, multivariate pattern analysis, machine learning approaches might increase the value of biomarkers in diagnostic and prognostic efforts. While several individual neuroimaging abnormalities of group-level significance have been reported in psychotic disorders using univariate analyses, they are of limited value for clinical purposes. In recent years, pattern classification algorithms such as support vector machines have been applied with some success for diagnosis, treatment response, and outcome prediction [63•, 64]. Machine learning approaches typically use algorithms to develop classifiers using a training set, and the classifiers are then used to test an entirely new imaging dataset. However, such approaches are yet to be used in routine, clinical settings. 
Fourth, the search for biomarkers needs to keep pace with the continual revisions in our evolving etiopathological models of psychotic disorders. For example, there is increasing evidence in support of the view that at least a subgroup of psychotic illnesses might be related to neuroinflammatory processes. Such inflammation may be reflected in microglial activation, which can be quantified in vivo using (R)-[(11)C]PK11195 and PET. Indeed, a small study showed that the binding potential of this ligand was increased in total gray matter in the early course of schizophrenia [65]. Another putative biomarker for brain neuroinflammation is extracellular water as measured by DTI; a recent study has shown a significant increase in the extracellular volume suggesting impaired axonal integrity related to brain inflammation in early course schizophrenia [66]. Another pathophysiological mechanism implicated in schizophrenia is oxidative stress. Glutathione, an antioxidant, can be measured using ${ }^{1} \mathrm{H}$ MRS and has been found to be elevated in patients in the early course of schizophrenia [67], suggesting that this may be a valuable biomarker. Each such imaging measure may reflect a specific aspect of the disease pathophysiology and may therefore serve as biomarkers to predict treatment response to the relevant treatment, e.g., using an anti-inflammatory or antioxidant treatment. Candidates for such biomarkers may be derived from preclinical data, e.g., animal models [68]. Translational discovery needs to be bi-directional, and human imaging data can potentially be modeled in animals. For example, studies of intrinsic brain connectivity using restingstate fMRI, which does not require animals to perform tasks, can be investigated across species and disease states [69•]. While such research is still in its infancy, these approaches hold promise for the future development of translational biomarkers.

Finally, even if one or more biomarkers are found to be of clinical relevance, that does not necessarily mean applicability in the real world. There is currently a lack of cohesion in the methods used to obtain and analyze all forms of imaging data. Standardized methods of image acquisition and postprocessing will be needed. Biomarkers must be determined objectively. Large multisite studies across diverse clinical settings will be needed to reduce variability and subjectivity of image analysis. Increasing automation of data processing and more clear validation of quantitative imaging data by clinically relevant measures such as outcome, treatment response, and association with etiological (such as genomic) data will also be needed $[70 \bullet \cdot$. Combined use of clinical and imaging biomarker data for prediction questions is already yielding promising results in other neuropsychiatric disorders. Data from the Alzheimer's Disease Neuroimaging initiative (ADNI) show that a scale combining cognitive and sMRI data (such as hippocampal and middle temporal thinning) had $91 \%$ power to predict conversion from amnestic mild cognitive impairment to Alzheimer's disease $[71 \bullet \cdot]$. In this context, observations of cortical thinning in CHR subjects who convert to psychosis during follow-up [72] are of importance. Hopefully, systematic evaluation of such leads in large, multisite trials will yield clinically useful biomarkers for psychotic disorders in the near future.

Acknowledgments M.K. receives support from MH78113. Thank you to Pratik Bahekar for his assistance with the literature search.

\section{Compliance with Ethics Guidelines}

Conflict of Interest Brandon Hager and Matcheri Keshavan have no relevant disclosures.

Human and Animal Rights and Informed Consent This article does not contain any studies with human or animal subjects performed by the author.

\section{References}

Papers of particular interest, published recently, have been highlighted as:

- Of importance

- Of major importance

1. Biomarkers Definitions Working Group. Biomarkers and surrogate endpoints: preferred definitions and conceptual framework. Clin Pharmacol Ther. 2001;69:89-95.

2.• Prata D, Mechelli A, Kapur S. Clinically meaningful biomarkers for psychosis: a systematic and quantitative review. Neurosci Biobehav Rev. 2014;45:134-41. A systematic look at the value of biomarkers for clinical psychiatry. The results are sobering, with only one biomarker possibly meeting the stringent criteria for a clinically useful biomarker.

3. Keshavan MS, Tandon R, Boutros NN, Nasrallah HA. Schizophrenia, "just the facts": what we know in 2008 Part 3: neurobiology. Schizophr Res. 2008;106(2-3):89-107.

4. Cochrane Collaboration. Cochrane Handbook for Systematic Reviews of Interventions. 2008. Available from: http://www. cochrane-handbook.org/.

5. Nakagawa S, Cuthill IC. Effect size, confidence interval and statistical significance: a practical guide for biologists. Biol Rev Camb Philos Soc. 2007;82(4):591-605.

6. Pantelis C, Yücel M, Wood SJ, et al. Structural brain imaging evidence for multiple pathological processes at different stages of brain development in schizophrenia. Schizophr Bull. 2005;31(3): 672-96.

7. Shepherd A, Laurens K, Matheson S, Carr V, Green M. Systematic meta-review and quality assessment of the structural brain alterations in schizophrenia. Neurosci Biobehav Rev. 2012;36:134256. An exhaustive meta-review synthesizing the available information from systematic reviews of structural alterations in both chronic and first-episode schizophrenia.

8. Bora E, Fornito A, Radua J, Walterfang M, Seal M, Wood SJ, et al. Neuroanatomical abnormalities in schizophrenia: a multimodal voxelwise meta-analysis and meta-regression analysis. Schizophr Res. 2011;127(1-3):46-57.

9. Shea BJ, Bouter LM, Peterson J, et al. External validation of a measurement tool to assess systematic reviews (AMSTAR). PLoS ONE. 2007;2(12):e1350. 
10. Shea BJ, Hamel C, Wells GA, et al. AMSTAR is a reliable and valid measurement tool to assess the methodological quality of systematic reviews. J Clin Epidemiol. 2009;62(10):1013-20.

11. Higgins JP, Thompson SG, Deeks JJ, Altman DG. Measuring inconsistency in meta-analyses. BMJ. 2003;327(7414):557-60.

12. Cohen J. Statistical power analysis for the behavioural sciences. 2nd ed. Hillsdale: Lawrence Erlbaum Associates; 1988.

13. Hedges L, Holkin I. Statistical methods for meta-analysis. New York: Academic Press; 1985.

14. Egger M, Davey Smith G, Schneider M, Minder C. Bias in metaanalysis detected by a simple, graphical test. BMJ. 1997;315:62934.

15. Haijma SV, Van Haren N, Cahn W, Koolschijn PC, Hulshoff Pol HE, Kahn RS. Brain volumes in schizophrenia: a meta-analysis in over 18000 subjects. Schizophr Bull. 2013;39(5):1129-38.

16. Adriano F, Spoletini I, Caltagirone C, Spalletta G. Updated metaanalyses reveal thalamus volume reduction in patients with firstepisode and chronic schizophrenia. Schizophr Res. 2010;123(1): $1-14$.

17. Adriano F, Caltagirone C, Spalletta G. Hippocampal volume reduction in first-episode and chronic schizophrenia: a review and metaanalysis. Neurosci: Rev J Bringing Neurobiol Neurol Psychiatry. 2012;18(2):180-200.

18. Vita A, De Peri L, Silenzi C, Dieci M. Brain morphology in firstepisode schizophrenia: a meta-analysis of quantitative magnetic resonance imaging studies. Schizophr Res. 2006;82(1):75-88.

19. De Peri L, Crescini A, Deste G, Fusar-poli P, Sacchetti E, Vita A. Brain structural abnormalities at the onset of schizophrenia and bipolar disorder: a meta-analysis of controlled magnetic resonance imaging studies. Curr Pharm Des. 2012;18(4):486-94.

20. Boos HB, Aleman A, Cahn W, Hulshoff Pol H, Kahn RS. Brain volumes in relatives of patients with schizophrenia: a meta-analysis. Arch Gen Psychiatry. 2007;64(3):297-304.

21. Arnone D, Cavanagh J, Gerber D, Lawrie S, Ebmeier K, Mcintosh A. Magnetic resonance imaging studies in bipolar disorder and schizophrenia: meta-analysis. Br J Psychiatry. 2009;195:194-201.

22. Kempton MJ, Geddes JR, Ettinger U, Williams SC, Grasby PM. Meta-analysis, database, and meta-regression of 98 structural imaging studies in bipolar disorder. Arch Gen Psychiatry. 2008;65(9): 1017-32.

23. Arnone D, Mcintosh AM, Tan GM, Ebmeier KP. Meta-analysis of magnetic resonance imaging studies of the corpus callosum in schizophrenia. Schizophr Res. 2008;101(1-3):124-32.

24. Ivleva EI, Bidesi AS, Keshavan MS, et al. Gray matter volume as an intermediate phenotype for psychosis: Bipolar-Schizophrenia Network on Intermediate Phenotypes (B-SNIP). Am J Psychiatry. 2013;170(11):1285-96.

25. Vita A, Peri L, Deste G, Sacchetti E. Progressive loss of cortical gray matter in schizophrenia: a meta-analysis and meta-regression of longitudinal MRI studies. Transl Psychiatry. 2012;2:E190.

26. Olabi B, Ellison-Wright I, McIntosh AM, Wood SJ, Bullmore E, Lawrie SM. Are there progressive brain changes in schizophrenia? A meta-analysis of structural magnetic resonance imaging studies. Biol Psychiatry. 2011;70:88-96.

27. Fusar-Poli P, Smieskova R, Kempton M, Ho B, Andreasen N, Borgwardt S. Progressive brain changes in schizophrenia related to antipsychotic treatment? A meta-analysis of longitudinal MRI studies. Neurosci Biobehav Rev. 2013;37:1680-91.

28. Kempton MJ, Stahl D, Williams SCR, DeLisi LE. Progressive lateral ventricular enlargement in schizophrenia: a meta-analysis of longitudinal MRI studies. Schizophr Res. 2010;120(1-3):54-62.

29. Fusar-Poli P, Meyer-Lindenberg A. Striatal presynaptic dopamine in schizophrenia, part ii: meta-analysis of [18 F/11C]-DOPA PET studies. Schizophr Bull. 2012;33-42.

30. Howes O, Kambeitz J, Kim E, Stahl D, Slifstein M, Abi-Dargham A, et al. The nature of dopamine dysfunction in schizophrenia and what this means for treatment. Arch Gen Psychiatry. 2012;69(8): 776-86.

31. Kraguljac NV, Reid M, White D, et al. Neurometabolites in schizophrenia and bipolar disorder - a systematic review and meta-analysis. Psychiatry Res. 2012;203(2-3):111-25.

32. Brugger S, Davis J, Leucht S, Stone J. Proton magnetic resonance spectroscopy and illness stage in schizophrenia - a systematic review and meta-analysis. Biol Psychiatry. 2011;69:495-503.

33. Marsman A, Heuvel M, Klomp D, Kahn R, Luijten P, Pol H. Glutamate in schizophrenia: a focused review and meta-analysis of 1H-MRS studies. Schizophr Bull. 2013;39(1):120-9.

34. Mondino M, Brunelin J, Saoud M. N-acetyl-aspartate level is decreased in the prefrontal cortex in subjects at-risk for schizophrenia. Front Psychiatry. 2013;4:99.

35. Patel S, Mahon K, Wellington R, Zhang J, Chaplin W, Szeszko PR. A meta-analysis of diffusion tensor imaging studies of the corpus callosum in schizophrenia. Schizophr Res. 2011;129(2-3):149-55.

36. Ellison-wright I, Bullmore E. Meta-analysis of diffusion tensor imaging studies in schizophrenia. Schizophr Res. 2009;108(1-3):310.

37. Kuswanto CN, Teh I, Lee TS, Sim K. Diffusion tensor imaging findings of white matter changes in first episode schizophrenia: a systematic review. Clin Psychopharmacol Neurosci. 2012;10(1): $13-24$.

38. Minzenberg MJ, Laird AR, Thelen S, Carter CS, Glahn DC. Metaanalysis of 41 functional neuroimaging studies of executive function in schizophrenia. Arch Gen Psychiatry. 2009;66(8):811-22.

39. Macdonald AW, Thermenos HW, Barch DM, Seidman LJ. Imaging genetic liability to schizophrenia: systematic review of FMRI studies of patients' nonpsychotic relatives. Schizophr Bull. 2009;35(6): $1142-62$.

40. Smieskova R, Marmy J, Schmidt A, et al. Do subjects at clinical high risk for psychosis differ from those with a genetic high risk? a systematic review of structural and functional brain abnormalities. Curr Med Chem. 2013;20(3):467-81.

41. Taylor SF, Kang J, Brege IS, Tso IF, Hosanagar A, Johnson TD. Meta-analysis of functional neuroimaging studies of emotion perception and experience in schizophrenia. Biol Psychiatry. 2012;71(2):136-45.

42. Fusar-poli P. Voxel-wise meta-analysis of fMRI studies in patients at clinical high risk for psychosis. J Psychiatry Neurosci. 2012;37(2):106-12.

43. Cooper D, Barker V, Radua J, Fusar-poli P, Lawrie SM. Multimodal voxel-based meta-analysis of structural and functional magnetic resonance imaging studies in those at elevated genetic risk of developing schizophrenia. Psychiatry Res. 2014;221(1):69-77.

44. Radua J, Borgwardt S, Crescini A, et al. Multimodal meta-analysis of structural and functional brain changes in first episode psychosis and the effects of antipsychotic medication. Neurosci Biobehav Rev. 2012;36(10):2325-33.

45. Jardri R, Pouchet A, Pins D, Thomas P. Cortical activations during auditory verbal hallucinations in schizophrenia: a coordinate-based meta-analysis. Am J Psychiatry. 2011;168(1):73-81.

46. Fusar-poli P, Broome MR, Woolley JB, et al. Altered brain function directly related to structural abnormalities in people at ultra high risk of psychosis: longitudinal VBM-fMRI study. J Psychiatr Res. 2011;45(2):190-8.

47. Tamminga CA, Pearlson G, Keshavan M, Sweeney J, Clementz B, Thaker G. Bipolar and schizophrenia network for intermediate phenotypes: outcomes across the psychosis continuum. Schizophr Bull. 2014;40 Suppl 2:S131-7.

48. Hyman SE. Can neuroscience be integrated into the DSM-V? Nat Rev Neurosci. 2007;8(9):725-32.

49. Velakoulis D, Wood SJ, Wong MT, McGorry PD, Yung A, Phillips L, et al. Hippocampal and amygdala volumes according to psychosis stage and diagnosis: a magnetic resonance imaging study of 
chronic schizophrenia, first-episode psychosis, and ultra-high-risk individuals. Arch Gen Psychiatry. 2006;63(2):139-49.

50. Thermenos HW, Keshavan MS, Juelich RJ, et al. A review of neuroimaging studies of young relatives of individuals with schizophrenia: a developmental perspective from schizotaxia to schizophrenia. Am J Med Genet B Neuropsychiatr Genet. 2013;162B(7): 604-35.

51. Cannon TD, Chung Y, He G, et al. Progressive reduction in cortical thickness as psychosis develops: a multisite longitudinal neuroimaging study of youth at elevated clinical risk. Biol Psychiatry. 2014. doi:10.1016/j.biopsych.2014.05.023.

52. Mattai AA, Weisinger B, Greenstein D, et al. Normalization of cortical gray matter deficits in nonpsychotic siblings of patients with childhood-onset schizophrenia. J Am Acad Child Adolesc Psychiatry. 2011;50(7):697-704.

53. Mcgorry P, Keshavan M, Goldstone S, et al. Biomarkers and clinical staging in psychiatry. World Psychiatry. 2014;13(3):211-23. A proposal of a new clinical staging model for severe mental disorders and the ways in which biomarkers could be integrated into such a framework.

54. Fusar-poli P, Howes OD, Allen P, et al. Abnormal frontostriatal interactions in people with prodromal signs of psychosis: a multimodal imaging study. Arch Gen Psychiatry. 2010;67(7):683-91.

55. Gottesman II, Gould TD. The endophenotype concept in psychiatry: ety-mology and strategic intentions. Am J Psychiatry. 2003;160:636-45.

56. Prasad KM, Keshavan MS. Structural cerebral variations as useful endophenotypes in schizophrenia: do they help construct "extended endophenotypes"? Schizophr Bull. 2008;34(4):774-90.

$57 . \bullet$ Thompson PM, Stein JL, Medland SE, et al. The ENIGMA Consortium: large-scale collaborative analyses of neuroimaging and genetic data. Brain Imaging Behav. 2014;8(2):153-82. A review of the current progress of the ENIGMA Consortium, a collaborative network of researchers working together on a range of large-scale studies that integrate neuroimaging and genetic data from 70 institutions worldwide.

58. DC V, Smith SM, Barch DM, et al. The WU-Minn Human Connectome Project: an overview. Neuroimage. 2013;80:62-79. A review of the systematic effort to map macroscopic human brain circuits, and the progress made in refining the methods for data acquisition and analysis.

59. Cocchi L, Harding IH, Lord A, Pantelis C, Yucel M, Zalesky A. Disruption of structure-function coupling in the schizophrenia connectome. Neuroimage Clin. 2014;4:779-87. A study with results supporting the core nature of fronto-striatal, fronto-thalamic, and fronto-temporal abnormalities in the schizophrenia connectome.

60. Keshavan MS, Clementz BA, Pearlson GD, Sweeney JA, Tamminga CA. Reimagining psychoses: an agnostic approach to diagnosis. Schizophr Res. 2013;146(1-3):10-6. A review of the evolution, current status and the constraints posed by traditional symptom-based diagnostic approaches in psychiatry, and a proposal to develop a biomarker-based approach.

61. Jack CR, Lowe VJ, Weigand SD, et al. Serial PIB and MRI in normal, mild cognitive impairment and Alzheimer's disease: implications for sequence of pathological events in Alzheimer's disease.
Brain. 2009;132(Pt 5):1355-65. An article attempting to utilize neuroimaging methods to identify biomarkers for Alzheimer's disease.

62. Kempton MJ, Mcguire P. How can neuroimaging facilitate the diagnosis and stratification of patients with psychosis?. Eur Neuropsychopharmacol. 2014. An article reviewing the current progress of utilizing neuroimaging methodologies to identify biomarkers for psychosis.

63. Orrù G, Pettersson-yeo W, Marquand AF, Sartori G, Mechelli A. Using support vector machine to identify imaging biomarkers of neurological and psychiatric disease: a critical review. Neurosci Biobehav Rev. 2012;36(4):1140-52. The article provides a useful overview of the applications of machine based learning to imaging biomarkers in neuropsychiatry.

64. Koutsouleris N, Meisenzahl EM, Davatzikos C, et al. Use of neuroanatomical pattern classification to identify subjects in at-risk mental states of psychosis and predict disease transition. Arch Gen Psychiatry. 2009;66(7):700-12.

65. BN V, Bossong MG, Boellaard R, et al. Microglia activation in recent-onset schizophrenia: a quantitative (R)-[11C]PK11195 positron emission tomography study. Biol Psychiatry. 2008;64(9):820 2.

66. Pasternak O, Westin CF, Bouix S, et al. Excessive extracellular volume reveals a neurodegenerative pattern in schizophrenia onset. J Neurosci. 2012;32(48):17365-72.

67. Wood SJ, Berger GE, Wellard RM, et al. Medial temporal lobe glutathione concentration in first episode psychosis: a 1H-MRS investigation. Neurobiol Dis. 2009;33(3):354-7.

68. Kellendonk C, Simpson EH, Kandel ER. Modeling cognitive endophenotypes of schizophrenia in mice. Trends Neurosci. 2009;32(6):347-58.

69. Smucny J, Wylie KP, Tregellas JR. Functional magnetic resonance imaging of intrinsic brain networks for translational drug discovery. Trends Pharmacol Sci. 2014;35(8):397-403. This article shows how intrinsic networks may show similar abnormalities in human diseases and animal models of these diseases, supporting their use as biomarkers in drug development in schizophrenia.

70.• Abramson RG, Burton KR, Yu JP, et al. Methods and challenges in quantitative imaging biomarker development. Acad Radiol. 2015;22(1):25-32. A discussion of the motivations for advancing quantitative imaging as a tool for biomarker research and development.

71.• Barnes DE, Cenzer IS, Yaffe K, Ritchie CS, Lee SJ. A point-based tool to predict conversion from mild cognitive impairment to probable Alzheimer's disease. Alzheimers Dement. 2014;10(6):646-55. Development of a novel point-based risk score combining functional dependence, cerebral MRI measures, and neuropsychological test scores which provided good accuracy for prediction of conversion from amnestic mild cognitive impairment to Alzheimer's disease.

72. Cannon TD, Chung Y, He G, et al. Progressive reduction in cortical thickness as psychosis develops: a multisite longitudinal neuroimaging study of youth at elevated clinical risk. Biol Psychiatry. 2015;77(2):147-57. 